\title{
The Number of Solutions of a Nonlinear Two Point Boundary Value Problem
}

\author{
THEODORE LAETSCH*
}

\author{
Communicated by J. B. Keller
}

1. Certain physical problems involving the steady state temperature distribution in a material bounded by two infinite parallel planes lead to the problem of determining those positive numbers $\lambda$ for which the boundary value problem

$$
u^{\prime \prime}(t)+\lambda f(u(t))=0, \quad 0 \leqq t \leqq 1, \quad u(0)=u(1)=0,
$$

has a positive solution $u(t)$. Here $f$ is a given function characteristic of the material, and $f(w)>0$ for all $w>0[2,4,6]$. Some properties of the positive solutions of (1.1) have been given by Joseph [5] and by Keller and Cohen [7].

The purpose of this paper is to obtain information on the values of $\lambda$ for which (1.1) has positive solutions, and to determine the behavior of the solutions as $\lambda$ varies (see Theorems 3.1 and 3.2). One of the principal results is that when $f(w)$ is a convex function of $w$, with $f(0)>0$, then (1.1) has, for each $\lambda>0$, either zero, one, or two positive solutions on the interval $(0,1)$. For such $f$, we shall determine a number $\mu_{1} \geqq 0$ and prove the existence of a number $\lambda^{*} \geqq \mu_{1}$ such that, if $\mu_{1}=0$, then $\lambda^{*}>0$ and (1.1) has two solutions for $0<\lambda<\lambda^{*}$, one for $\lambda=\lambda^{*}$, and none for $\lambda>\lambda^{*}$; if $0<\mu_{1}<\lambda^{*}$, then (1.1) has one solution for $0<\lambda \leqq \mu_{1}$ and $\lambda=\lambda^{*}$, two for $\mu_{1}<\lambda<\lambda^{*}$, and none for $\lambda>\lambda^{*}$; if $0<$ $\mu_{1}=\lambda^{*}<+\infty$, then (1.1) has one solution for $0<\lambda<\lambda^{*}$ and none for $\lambda \geqq \lambda^{*}$; and if $\mu_{1}=+\infty$, then $\lambda^{*}=+\infty$ and (1.1) has solutions for all $\lambda>0$. We shall give a necessary and sufficient condition for $\lambda^{*}>\mu_{1}$.

The first step in our analysis of (1.1) will be to reduce the problem of solving (1.1) to a quadrature. This will give an explicit expression for the values of $\lambda$ for which (1.1) has solutions as a function of the maximum value $\|u\|$ of the corresponding solution $u(t)$ of (1.1), and information concerning the behavior of the maximum $\|u\|$ of the solutions of (1.1) as $\lambda$ varies is obtained from this expression. The results concerning the number of solutions when $f$ is convex are obtained directly from (1.1).

- This research was done while the author held a National Defense Education Act Title IV Fellowship. Portions of this paper are contained in a thesis submitted to the California Institute of Technology in partial fulfillment of the requirements for the degree of Doctor of Philosophy. 
2. We consider the nonlinear eigenvalue problem (1.1), where all functions $f$ considered in connection with (1.1) are assumed to be non-negative continuous functions on the interval $[0, r)$ for some $r, 0<r \leqq+\infty$, with $f(w)>0$ for $0<w<r$. We wish to determine those values of $\lambda$ ("eigenvalues") for which (1.1) has non-negative not identically zero solutions (we shall refer to these briefly as positive solutions) and to determine the behavior of these solutions as functions of $\lambda$. Since we are interested only in positive solutions of (1.1), we assume $f$ is extended to a continuous non-negative function on $(-\infty, r)$. We may assume $\lambda>0$, since if $\lambda \leqq 0$, then no solution of (1.1) may have a positive maximum on $(0,1)$ and therefore (1.1) has no positive solutions. On the other hand, all nonzero solutions of (1.1) for $\lambda>0$ are strictly positive and have exactly one maximum on $(0,1)[13$, Chapter 1$]$.

Let $t_{0}$ be the point at which some solution $u(t)$ of (1.1) assumes its maximum $\|u\|=u\left(t_{0}\right)$. Then $u^{\prime}(t) \geqq 0$ on $\left[0, t_{0}\right]$ and $u^{\prime}(t) \leqq 0$ on $\left[t_{0}, 1\right]$. Thus (1.1) may be integrated as follows: Let $F(w)=\int_{0}^{w} f(v) d v$; then

$$
\begin{aligned}
& \frac{1}{2} u^{\prime}(t)^{2}+\lambda F(u(t))=\lambda F(\|u\|), \\
& t(2 \lambda)^{1 / 2}=\int_{0}^{u(t)} \frac{d w}{[F(\|u\|)-F(w)]^{1 / 2}}, \quad 0 \leqq t \leqq t_{0}, \\
& \quad(1-t)(2 \lambda)^{1 / 2}=\int_{0}^{u(t)} \frac{d w}{[F(\|u\|)-F(w)]^{1 / 2}}, \quad t_{0} \leqq t \leqq 1 .
\end{aligned}
$$

Setting $t=t_{0}$ and $u(t)=\|u\|$, we see that $t_{0}=\frac{1}{2}$ and $u(t)=u(1-t)$; that is, any solution of (1.1) is symmetric about the point $t=\frac{1}{2}$. The problem (1.1) is therefore equivalent to

$$
u^{\prime \prime}(t)+\lambda f(u(t))=0, \quad 0 \leqq t \leqq \frac{1}{2}, \quad u(0)=u^{\prime}\left(\frac{1}{2}\right)=0 .
$$

Joseph [5] has studied an equivalent problem with the boundary conditions $u^{\prime}(0)=u(1)=0$.

The equations (2.2) may be used to construct the solutions of (1.1) or (2.3). For any number $\rho \varepsilon(0, r)$, define $\lambda$ by

$$
\lambda^{1 / 2}=2^{1 / 2} \int_{0}^{\rho} \frac{d w}{[F(\rho)-F(w)]^{1 / 2}}=2^{1 / 2} \rho \int_{0}^{1} \frac{d v}{[F(\rho)-F(\rho v)]^{1 / 2}}
$$

(cf. (2.2)). Then the equation

$$
t(2 \lambda)^{1 / 2}=\int_{0}^{u} \frac{d w}{[F(\rho)-F(w)]^{1 / 2}}
$$

defines a one-to-one relation between $t$ and $u$ for $0 \leqq t \leqq \frac{1}{2}$ and $0 \leqq u \leqq \rho$ such that $t=0$ iff $u=0$ and $t=\frac{1}{2}$ iff $u=\rho$. The function $u(t)$ so defined is easily seen to be twice differentiable and to satisfy (2.3).

The integral in (2.4) is improper at the upper limit $w=\rho$. Near this limit, $F$ behaves like $F(w)=F(\rho)+f(\rho)(w-\rho)+o(w-\rho)$, so the integral is convergent if $f(\rho)>0$. The last integral in (2.4) can be written as 


$$
\int_{0}^{1 / 2} \frac{d v}{[F(\rho)-F(\rho v)]^{1 / 2}}+\int_{1 / 2}^{1} \frac{d v}{[F(\rho)-F(\rho v)]^{1 / 2}}
$$

the first integral here clearly defines a continuous function of $\rho$ for $0<\rho<r$, while the fact that $f(w)$ has a positive minimum on any closed subinterval of $(0, r)$ implies that the second integral is uniformly convergent and hence defines a continuous function of $\rho$ for $0<\rho<r$. Thus we obtain:

Theorem 2.1. For any number $\rho \varepsilon(0, r)$, there exist exactly one number $\lambda_{f}\{\rho\}$ and one (non-negative) function $u\{\rho\}$ on $[0,1]$ which satisfy $(1.1)$ and $\|u\{\rho\}\|=\rho$; $\lambda_{f}\{\rho\}$ is a continuous function of $\rho$.

The next result shows that, for a fixed $\lambda$, different solutions of (1.1) must take on different values at all points of the interval $(0,1)$. This result is critical for the proofs of Lemmas 3.1 and 3.2 below.

Theorem 2.2. Let $u_{1}$ and $u_{2}$ be two distinct solutions of (1.1) for a fixed $\lambda>0$. Then either $u_{1}(t)<u_{2}(t)$ for all $t \varepsilon(0,1)$, or $u_{2}(t)<u_{1}(t)$ for all $t \varepsilon(0,1)$.

Proof. By Theorem 2.1, $\left\|u_{1}\right\| \neq\left\|u_{2}\right\|$; suppose $\left\|u_{1}\right\|<\left\|u_{2}\right\|$. Since $F$ is strictly increasing, $F\left(\left\|u_{1}\right\|\right)<F\left(\left\|u_{2}\right\|\right)$. Since $u_{1}^{\prime}(0) \neq u_{2}^{\prime}(0)$ (by equation (2.1)), either $u_{1}(t)<u_{2}(t)$ for $0<t \leqq \frac{1}{2}$, or there is a smallest number $c, 0<c<\frac{1}{2}$, such that $u_{1}(c)=u_{2}(c)$; but this contradicts (2.2) (with $t_{0}=\frac{1}{2}$ ). Thus $u_{1}(t)<$ $u_{2}(t)$ for $0<t \leqq \frac{1}{2}$, and by symmetry $u_{1}(t)<u_{2}(t)$ for $0<t<1$. Q.E.D.

The following comparison theorem is obvious from equation (2.4):

Theorem 2.3. Suppose that $f_{1}(w) \leqq f_{2}(w)$ for $w \varepsilon[0, r)$. Then $\lambda_{f_{1}}\{\rho\} \geqq \lambda_{f_{1}}\{\rho\}$ for $0<\rho<r$. If, additionally, $f_{1}\left(w_{0}\right)<f_{2}\left(w_{0}\right)$ for some $w_{0} \varepsilon[0, r)$, then $\lambda_{f_{1}}\{\rho\}>$ $\lambda_{f,}\{\rho\}$ for $w_{0}<\rho<r$.

Various theorems concerning the continuous dependence of the eigenvalues $\lambda_{f}\{\rho\}$ on the function $f$ in equation (1.1) can be obtained from the representation (2.4) for $\lambda_{f}\{\rho\}$; we mention without proof the following simple result:

Theorem 2.4. Let $f, g, f_{1}, f_{2}, f_{8}, \cdots$ be continuous functions on $[0, r), 0<$ $\boldsymbol{r} \leqq+\infty$, which are positive on $(0, r)$, and let $h$ be locally integrable on $(0, r)$. Suppose that $\left\{f_{n}(w)\right\}$ converges to $f(w)$ almost everywhere on $(0, r)$ and that $g(w) \leqq$ $f_{n}(w) \leqq h(w)$ for $0 \leqq w<r$. Then $\left\{\lambda_{f_{n}}\{\rho\}\right\}$ converges to $\left\{\lambda_{f}\{\rho\}\right\}$ for each $\rho \varepsilon(0, r)$.

The behavior of $\lambda_{s}\{\rho\}$ for large $\rho$ and for small $\rho$ may be obtained from equation (2.4) and the corresponding behavior of $f(w)$. Since the function $f$ is fixed in each of the following theorems, we write $\lambda\{\rho\}$ in place of $\lambda_{f}\{\rho\}$. We recall that $f$ is assumed to be continuous on $[0, r)$ and positive on $(0, r)$, with $0<$ $r \leqq+\infty$.

Theorem 2.5. Suppose that either $r<+\infty$ and $0<\lim _{\inf _{w \rightarrow r-}}(r-w)$. $f(w) \leqq+\infty$ or $r=+\infty$ and $\lim _{w \rightarrow+\infty} f(w) / w=+\infty$. Then $\lim _{\rho \rightarrow r} \lambda\{\rho\}=0$. 
Proof. If $r=+\infty$, then given any number $M>0$, there is a positive number $R$ such that $f(w)>M w$ for $R \leqq w$. Thus, if $2 R \leqq \rho$, then

$$
F(\rho)-F(w)=\int_{w}^{\rho} f(v) d v \geqq \frac{1}{2} M\left(\rho^{2}-w^{2}\right)
$$

for $R \leqq w<\rho$, and

$$
F(\rho)-F(w)=\int_{R}^{\rho}+\int_{w}^{R} f(v) d v \geqq \frac{1}{2} M\left(\rho^{2}-R^{2}\right) \geqq \frac{3}{2} M R^{2}
$$

for $0 \leqq w \leqq R$. From equation (2.4), whenever $2 R \leqq \rho$,

$$
\begin{aligned}
{\left[\frac{1}{2} \lambda\{\rho\}\right]^{1 / 2}=\int_{0}^{\rho} \frac{d w}{[F(\rho)-F(w)]^{1 / 2}} } & \leqq\left(\frac{2}{M}\right)^{1 / 2}\left\{\int_{R}^{\rho} \frac{d w}{\left(\rho^{2}-w^{2}\right)^{1 / 2}}+\int_{0}^{R} \frac{d w}{\left(3 R^{2}\right)^{1 / 2}}\right\} \\
& \leqq\left(\frac{2}{M}\right)^{1 / 2}\left\{\frac{\pi}{2}+\frac{1}{3^{1 / 2}}\right\}
\end{aligned}
$$

Hence $\lim _{\rho \rightarrow+\infty} \lambda\{\rho\}=0$.

If $r<+\infty$, there are positive numbers $R<r$ and $k$ such that $f(w) \geqq k^{2} /(r-w)$ for $R \leqq w<r$. Thus if $R<\rho<r$, equation (2.4) implies that

$$
\begin{aligned}
{\left[\frac{1}{2} \lambda\{\rho\}\right]^{1 / 2} \leqq } & \int_{0}^{R}\left(k^{2} \ln \frac{r-R}{r-\rho}\right)^{-1 / 2} d w+\int_{R}^{\rho}\left(k^{2} \ln \frac{r-w}{r-\rho}\right)^{-1 / 2} d w \\
& =k^{-1}\left\{R[\xi(\rho)]^{-1 / 2}+(r-\rho) \int_{0}^{\xi(\rho)} x^{-1 / 2} e^{x} d x\right\} \rightarrow 0 \text { as } \rho \rightarrow r-,
\end{aligned}
$$

where $\xi(\rho) \equiv \ln (r-R)-\ln (r-\rho)$. This completes the proof.

The following theorem is proved similarly:

Theorem 2.6. Suppose that either $r<+\infty$ and $0 \leqq \lim \sup _{w \rightarrow r-} f(w) /(r-$ $w)<+\infty$ or $r=+\infty$ and $\lim _{w \rightarrow+\infty} f(w) / w=0$. Then $\lim _{\rho \rightarrow r} \lambda\{\rho\}=+\infty$.

If $f(w)$ has the asymptotic form $m w^{s}$ as $w \rightarrow+\infty$, then we are able to specify more precisely the asymptotic behavior of $\lambda\{\rho\}$ as $\rho \rightarrow+\infty$ :

Theorem 2.7. Suppose $r=+\infty$ and $f(w) \sim m w^{s}$ as $w \rightarrow+\infty$ for some real number $s$ and positive number $m$. Then $\lambda\{\rho\} \sim\left(\tau_{s}^{2} / m\right) \rho^{1-s}$ as $\rho \rightarrow+\infty$, where

$$
\tau_{s}= \begin{cases}2^{1 / 2} \int_{0}^{1}\left[\frac{1+s}{1-v^{1+s}}\right]^{1 / 2} d v, & s \neq-1 \\ 2^{1 / 2} \Gamma\left(\frac{1}{2}\right)=(2 \pi)^{1 / 2} & , s=-1 .\end{cases}
$$

In particular, if $\lim _{w \rightarrow+\infty} f(w) / w=m$, then $\lim _{\rho \rightarrow+\infty} \lambda\{\rho\}=\pi^{2} / m$. (Observe that $\pi^{2} / m$ is the first eigenvalue of the linear problem

$$
\begin{gathered}
\varphi^{\prime \prime}(t)+\mu m \varphi(t)=0, \quad 0 \leqq t \leqq 1, \\
\varphi(0)=\varphi(1)=0 .
\end{gathered}
$$

Cf. Krasnosel'skii [9, p. 208].) 
Proof. Simple algebraic transformations of equation (2.4) yield

$$
\begin{gathered}
{\left[\frac{1}{2} m \lambda\{\rho\}\right]^{1 / 2}=m^{1 / 2} \rho \int_{0}^{1} \frac{d v}{[F(\rho)-F(\rho v)]^{1 / 2}}=\rho^{(1-s) / 2}} \\
\cdot \int_{0}^{1}\left[\frac{1+s}{1-v^{1+s}}\right]^{1 / 2} d v-\rho^{(1-s) / 2} \int_{0}^{1}\left[\frac{1+s}{1-v^{1+s}}\right]^{1 / 2} \frac{[1+\gamma(\rho, v)]^{1 / 2}-1}{[1+\gamma(\rho, v)]^{1 / 2}} d v,
\end{gathered}
$$

for $s \neq-1$, where

$$
\gamma(\rho, v)=\frac{1+s}{m} \frac{\int_{\rho v}^{\rho}\left[f(w)-m w^{s}\right] d w}{\rho^{1+s}\left(1-v^{1+s}\right)} .
$$

We will show that the last integral in (2.5) converges to zero as $\rho \rightarrow+\infty$. Suppose first that $s>-1$. Since

$$
\lim _{v \rightarrow+\infty}\left[\frac{f(v)}{v^{s}}-m\right]=0
$$

we have for $0 \leqq v \leqq \frac{1}{2}$, using l'Hospital's Rule,

$$
\lim _{\rho \rightarrow+\infty}|\gamma(\rho, v)| \leqq \lim _{\rho \rightarrow+\infty} \frac{1+s}{m \rho^{1+s}\left(1-v^{1+s}\right)} \int_{0}^{\rho}\left|f(w)-m w^{s}\right| d s=0
$$

uniformly in $v$; for $\frac{1}{2} \leqq v \leqq 1$,

$$
\begin{aligned}
\lim _{\rho \rightarrow+\infty}|\gamma(\rho, v)| \leqq \lim _{\rho \rightarrow+\infty} \sup \left\{\left|\frac{f(w)-m w^{s}}{w^{s}}\right|: w \geqq \frac{1}{2} \rho\right\} & \\
& \cdot \frac{1+s}{m \rho^{1+s}\left(1-v^{1+s}\right)} \int_{\rho_{\nu}}^{\rho} w^{*} d w=0
\end{aligned}
$$

uniformly in $v$. It follows that the last integral in equation (2.5) converges to zero as $\rho \rightarrow+\infty$, and therefore equation (2.5) implies

$$
\lim _{\rho \rightarrow+\infty}\left[\frac{1}{2} m \rho^{s-1} \lambda\{\rho\}\right]^{1 / 2}=\int_{0}^{1}\left[\frac{1+s}{1-v^{1+s}}\right]^{1 / 2} d v
$$

for $s>-1$.

Suppose next that $s<-1$.

For any $R>0$ and $\rho \geqq 2 R$,

$$
|1+s| \frac{\int_{\rho v}^{\rho}\left|f(w)-m w^{*}\right| d w}{\rho^{1+s}\left|1-v^{1+s}\right|} \leqq \sup \left\{\left|\frac{f(w)}{w^{s}}-m\right|: w \geqq R\right\} \equiv \epsilon_{R}
$$

if $R / \rho \leqq v \leqq 1$. Similarly, $\gamma(\rho, v)$ can be bounded uniformly in $\rho$ and $v$ for $\rho \geqq 2 R$ and $0<v \leqq \frac{1}{4}$. Given $\epsilon>0$, we choose $R$ so large that

$$
\frac{\left(1+\epsilon_{R}\right)^{1 / 2}-1}{\left(1-\epsilon_{R}\right)^{1 / 2}} \int_{0}^{1} \frac{d v}{\left|1-v^{1+s}\right|^{1 / 2}}<\frac{\epsilon}{2}
$$


and then choose $R_{0} \geqq 4 R$ so large that

$$
\frac{R}{R_{0}} \sup \left\{\left|\left(\frac{1+s}{1-v^{1+8}}\right)^{1 / 2} \frac{[1+\gamma(\rho, v)]^{1 / 2}-1}{[1+\gamma(\rho, v)]^{1 / 2}}\right|: 0<v \leqq \frac{1}{4}, \quad \rho \geqq 2 R\right\} \leqq \frac{\epsilon}{2} .
$$

Then, if we write the last integral in (2.5) as the sum of an integral from 0 to $R / R_{0}$ and one from $R / R_{0}$ to 1 ,

$$
\int_{0}^{1}\left(\frac{1+s}{1-v^{1+\varepsilon}}\right)^{1 / 2} \frac{[1+\gamma(\rho, v)]^{1 / 2}-1}{[1+\gamma(\rho, v)]^{1 / 2}} d v \leqq \epsilon
$$

for $\rho \geqq R_{0}$. Thus equation (2.5) implies that equation (2.6) holds also for $s<-1$. The proof for $s=-1$ is similar.

Q.E.D.

Our next theorem obtains bounds on $\lambda\{\rho\}$ resulting from a comparison of $f$ with a linear function $m w$. The result is of particular interest if $m=$ $\lim _{w \rightarrow+\infty} f(w) / w$ (cf. Theorem 2.7 and Theorem 2.12).

Theorem 2.8. Let $m \varepsilon(0,+\infty)$. If $r=+\infty$ and there is a constant $\eta>0$ such that $f(w)-m w \geqq \eta w^{-1}$ (or $\left.\leqq-\eta w^{-1}\right)$ for all sufficiently large $w$, then $\lambda\{\rho\}<\pi^{2} / m$ (or $>\pi^{2} / m$, respectively) for all sufficiently large $\rho$. If $r \leqq+\infty$ and $f(w)-m w \geqq 0$ (or $\leqq 0)$ for all $w \varepsilon[0, r)$, then $\lambda\{\rho\} \leqq \pi^{2} / m$ (or $\geqq \pi^{2} / m$, respectively) for $0<\rho<r ; \lambda\{\rho\}=\pi^{2} / m$ for some $\rho>0$ if and only if $f(w)=m w$ for all $w \leqq \rho$.

Proof. If $f(w)-m w \geqq 0$ or $\leqq 0$ for all $w$, then the last sentence of the theorem follows from Theorem 2.3.

Suppose that $f(w)-m w \geqq \eta w^{-1}$ for $w \geqq R$. We will use equation (2.5) with $s=1$. Let $M_{R}=\max \{|f(w)-m w|: 0 \leqq w \leqq R\}$, and let $R^{\prime}=R \exp \left(R M_{R} / \eta\right)>R$. Then $\gamma(\rho, v)>0$ whenever $\rho>R^{\prime}, 0 \leqq v \leqq 1$; for, if $R / \rho \leqq v<1$, then

$$
\int_{\rho v}^{\rho}[f(w)-m w] d w \geqq \int_{\rho,}^{\rho} \eta w^{-1} d w=\eta \ln v^{-1},
$$

so

$$
\gamma(\rho, v) \geqq \frac{2}{m} \frac{\eta\left(\ln v^{-1}\right)}{\rho^{2}\left(1-v^{2}\right)}>0 \text { for } \quad R / \rho \leqq v<1 \text {, }
$$

while for

$$
\begin{gathered}
0 \leqq v \leqq R / \rho \\
\int_{\rho_{\nu}}^{\rho}[f(w)-m w] d w \geqq-\int_{0}^{R}|f(w)-m w| d w+\int_{R}^{\rho} \eta w^{-1} d w \\
\geqq-R M_{R}+\eta \ln (\rho / R),
\end{gathered}
$$

so

$$
\gamma(\rho, v)>\frac{2}{m} \frac{-R M_{R}+\eta \ln \left(R^{\prime} / R\right)}{\rho^{2}\left(1-v^{2}\right)}=0 .
$$


From equation (2.5) it follows that $\lambda\{\rho\}<\pi^{2} / m$ for $\rho>R^{\prime}$. The inequality $\lambda\{\rho\}>\pi^{2} / m$ for $\rho>R^{\prime}$ if $f(w)-m w \leqq-\eta w^{-1}$ for $w>R$ may be proved similarly.

Q.E.D.

Analogous results on the behavior of $\lambda\{\rho\}$ as $\rho \rightarrow 0$ may be obtained from the behavior of $f(w)$ as $w \rightarrow 0$; the proofs in this case are similar to (but even simpler than) those above.

Theorem 2.9. If $\lim _{w \rightarrow 0+} f(w) / w=+\infty$, then $\lim _{\rho \rightarrow 0} \lambda\{\rho\}=0$. If $\lim _{w \rightarrow 0+} f(w) / w=0$, then $\lim _{\rho \rightarrow 0} \lambda\{\rho\}=+\infty$.

Theorem 2.10. Suppose $f(w) \sim a w^{s}$ as $w \rightarrow 0$ for some $a>0, s \geqq 0$. Then $\lambda\{\rho\} \sim\left(\tau_{s}^{2} / a\right) \rho^{1-s}$ as $\rho \rightarrow 0$. In particular, if $f(0)>0$, then $\lim _{\rho \rightarrow 0} \lambda\{\rho\}=0$; if $f(0)=0$ and $f$ is differentiable from the right at 0 , then $\lim _{\rho \rightarrow 0} \lambda\{\rho\}=\pi^{2} / f_{R}^{\prime}(0)$ (where $f_{R}^{\prime}(0)$ is the right derivative of $f$ at 0 ). (Note that when $f_{R}^{\prime}(0)>0, \pi^{2} / f_{R}^{\prime}(0)$ is the first eigenvalue of the "variational" problem

$$
\varphi^{\prime \prime}(t)+\mu f_{R}^{\prime}(0) \varphi(t)=0, \quad 0 \leqq t \leqq 1, \quad \varphi(0)=\varphi(1)=0 .
$$

Cf. Krasnosel'skii [9, p. 196].)

Corollary 2.10.1. Suppose $f$ is differentiable from the right at the origin, $f(0)=0$, and $f(w)-w f_{R}^{\prime}(0)<0($ or $>0)$ for sufficiently small $w$. Then $\lim _{\rho \rightarrow 0} \lambda\{\rho\}$ $=\pi^{2} / f_{R}^{\prime}(0)$ and $\lambda\{\rho\}>\pi^{2} / f_{R}^{\prime}(0)$ (or $<\pi^{2} / f_{R}^{\prime}(0)$, respectively) for sufficiently small $\rho$.

Proof. Theorems 2.10 and 2.8 .

Note that the hypothesis of the corollary is satisfied if $f(w)$ is strictly concave with $f_{R}^{\prime}(0)<+\infty$ (or strictly convex, respectively) for sufficiently small $w$.

Theorem 2.11. Let $f$ be non-decreasing on $(0, r)$. Then $\lambda\{\rho\} \geqq 8 \rho / f(\rho)$ for $0<\rho<r$.

Proof. Since $f$ is non-decreasing, $F$ is convex and differentiable, and therefore

$$
F(\rho)-F(w) \leqq f(\rho)(\rho-w)
$$

for $0 \leqq w \leqq \rho<r$. Thus

$$
\begin{aligned}
& {[\lambda\{\rho\}]^{1 / 2}=2^{1 / 2} \rho \int_{0}^{1} \frac{d v}{[F(\rho)-F(\rho v)]^{1 / 2}}} \\
& \geqq\left[\frac{2 \rho}{f(\rho)}\right]^{1 / 2} \int_{0}^{1} \frac{d v}{[1-v]^{1 / 2}}=8^{1 / 2}\left[\frac{\rho}{f(\rho)}\right]^{1 / 2} \text {.Q.E.D. }
\end{aligned}
$$

Observe that if $f$ is non-decreasing on $(0, r)$, then Theorem 2.5 and the second part of Theorem 2.9 follow immediately from Theorem 2.11.

The following lemma collects some results on convex functions which will be useful in the sequel. The proof of these results may be found, e.g., in Bourbaki [1]. 
Lemma 2.1. Let $f$ be a convex function on $(0, r)$; i.e., for every $w_{1}, w_{2}$ in $(0, r)$ and every $\alpha \varepsilon[0,1]$.

$$
f\left(\alpha w_{1}+(1-\alpha) w_{2}\right) \leqq \alpha f\left(w_{1}\right)+(1-\alpha) f\left(w_{2}\right) .
$$

If for fixed $w_{1}, w_{2}$, equality holds in $(2.8)$ for some $\alpha \varepsilon(0,1)$, then equality holds for all $\alpha \varepsilon(0,1) . f$ is continuous on $(0, r)$, has a right derivative $f_{R}^{\prime}(w)$ and a left derivative $f_{L}^{\prime}(w)$ at each point $w \varepsilon(0, r)$, and is differentiable except possibly at countably many points of $(0, r)$. The derivatives $f_{R}^{\prime}$ and $f_{L}^{\prime}$ are non-decreasing on $(0, r)$, and $f(w)-w_{R}^{\prime}(w)$ is non-increasing on $(0, r)$. Either $f$ is non-increasing on $(0, r)$, or $f$ is non-decreasing on $(0, r)$, or there is a point $a \boldsymbol{\varepsilon}(0, r)$ such that $f$ is non-increasing on $(0, a)$ and non-decreasing on $(a, r)$. If $f(0)>0$, then either $f(w) / w$ is non-increasing for $w \boldsymbol{\varepsilon}(0, r)$ or there is a point $b \boldsymbol{\varepsilon}(0, r)$ such that $f(w) / w$ is non-increasing for $w \varepsilon(0, b)$ and non-decreasing for $w \varepsilon(b, r)$. If $f(0)=0$ and $f$ is non-negative, then $f(w) / w$ is non-decreasing for $w \boldsymbol{\varepsilon}(0, r)$.

Our next theorem uses Theorem 2.8 to show that for certain convex functions $f$, equation (1.1) will have at least two solutions for some values of $\lambda$; Lemma 3.2 below shows that there can be at most two solutions for all $\lambda$ when $f$ is convex.

Theorem 2.12. Let $f$ be a convex function on $(0,+\infty)$, and let $m=$ $\lim _{w \rightarrow+\infty} f(w) / w(\leqq+\infty)$. If $f(0)>0$ and $f(w) / w$ is eventually strictly increasing (equivalently, if $w f_{R}^{\prime}(w)-f(w)$ is eventually positive), then $\lambda\{\rho\}$ has a maximum $\lambda^{*}>\pi^{2} / m$ at some finite number $\rho=\rho^{*}>0$, and thus (1.1) has at least two solutions for each $\lambda$ such that $\pi^{2} / m<\lambda<\lambda^{*}$.

Proof. By Lemma 2.1, $m=\lim _{w \rightarrow+\infty} f(w) / w$ exists since $f$ is convex, and by Theorems 2.7 and $2.10, \lim _{\rho \rightarrow+\infty} \lambda\{\rho\}=\pi^{2} / m$ and $\lim _{\rho \rightarrow 0} \lambda\{\rho\}=0$. If $m=$ $+\infty$, the result follows immediately from the continuity of $\lambda\{\rho\}$. If $m<+\infty$, then $f(w)-m w$ is a convex function of $w$ which is negative for sufficiently large $w$, and therefore $f(w)-m w$ is decreasing for all $w$. It follows that there is an $\epsilon>0$ such that for sufficiently large $w, f(w)-m w \leqq-\epsilon$. From Theorem 2.8, $\lambda\{\rho\}>\pi^{2} / m$ for sufficiently large $\rho$. The continuity of $\lambda\{\rho\}$ implies that $\lambda\{\rho\}$ has a maximum $\lambda^{*}>\pi^{2} / m$, and thus there are two solutions for each $\lambda$ with $\pi^{2} / m<\lambda<\lambda^{*}$.

Q.E.D.

3. In this section we obtain some results on the maximum number of solutions of (1.1) for fixed $\lambda$. Important use is made of Theorem 2.2. Observe that if $f$ is differentiable, then the hypothesis of Lemma 3.1 that $f(w) / w$ is nonincreasing is equivalent to the inequality $f(w)-w f^{\prime}(w) \geqq 0$. This means that the tangent to the graph of $f(w)$ against $w$ always intersects the positive vertical (ordinate) axis. In particular, the hypothesis of Lemma 3.1 is satisfied if $f$ is concave. Theorem 3.1 should be compared with [8, Theorem 7.14] and [11], and Lemma 3.2 with [8, Lemma 6.3], [10], [12], and [3]. Note, however, that we do not assume that $f$ is monotonically increasing.

Lemma 3.1. Let $f(w) / w$ be a non-increasing function of $w$ for $0<w<r$. 
If (1.1) has positive solutions $u_{1}, u_{2}$ for some fixed $\lambda$, with $\max \left\{\left\|u_{1}\right\|,\left\|u_{2}\right\|\right\}<r$, then $u_{1}=u_{2}$ unless $f(w)=\left(\pi^{2} / \lambda\right) w$ for $0 \leqq w \leqq \max \left\{\left\|u_{1}\right\|,\left\|u_{2}\right\|\right\}$.

Proof. If $u_{1} \neq u_{2}$, then by Theorem 2.2 we can assume that $u_{1}(t)<u_{2}(t)$ for $0<t<1$ and therefore $f\left(u_{1}(t)\right) / u_{1}(t) \geqq f\left(u_{2}(t)\right) / u_{2}(t)$ for $0<t<1$. Then

$$
0=\int_{0}^{1}\left[u_{1}(t) u_{2}^{\prime \prime}(t)-u_{2}(t) u_{1}^{\prime \prime}(t)\right] d t=\lambda \int_{0}^{1} u_{1}(t) u_{2}(t)\left[\frac{f\left(u_{1}(t)\right)}{u_{1}(t)}-\frac{f\left(u_{2}(t)\right)}{u_{2}(t)}\right] d t .
$$

Since the integrand is non-negative and $u_{1}(t) u_{2}(t)>0$ for $0<t<1$, we must have $f\left(u_{1}(t)\right) / u_{1}(t)=f\left(u_{2}(t)\right) / u_{2}(t)$ for $0<t<1$. Let $m_{0}=f\left(\left\|u_{2}\right\|\right) /\left\|u_{2}\right\|$; we assert that $f(w) / w=m_{0}$ for $0<w \leqq\left\|u_{2}\right\|$. To see this, we note that since $f\left(\| u_{2}||\right) /\left\|u_{2}\right\|=f\left(\| u_{1}||\right) /\left\|u_{1}\right\|$ and $f(w) / w$ is decreasing, we must have $f(w) / w$ $=m_{0}$ for $\left\|u_{1}\right\| \leqq w \leqq\left\|u_{2}\right\|$. If $w_{0}$ is such that $0<w_{0}<\left\|u_{2}\right\|$ and $f\left(w_{0}\right) / w_{0}=m_{0}$, then there is a point $t_{0}, 0<t_{0}<1$, such that $w_{0}=u_{2}\left(t_{0}\right)$ and $f\left(u_{2}\left(t_{0}\right)\right) / u_{2}\left(t_{0}\right)=$ $f\left(u_{1}\left(t_{0}\right)\right) / u_{1}\left(t_{0}\right)$. Thus, $w_{0}$ is an interior point of the interval $\left[u_{1}\left(t_{0}\right),\left\|u_{2}\right\|\right]$ and $f(w) / w=m_{0}$ for all $w \varepsilon\left[u_{1}\left(t_{0}\right),\left\|u_{2}\right\|\right]$. This shows that the set $\left\{w: 0<w \leqq\left\|u_{2}\right\|\right.$, $\left.f(w) / w=m_{0}\right\}$ is both open and (by continuity) closed relative to the interval (0, $\left.\left\|u_{2}\right\|\right]$; thus, $f(w) / w=m_{0}$ for all $w \varepsilon\left(0,\left\|u_{2}\right\|\right]$ and $f(w)=m_{0} w$ for $0 \leqq w \leqq$ $\left\|u_{2}\right\|$. Then $u_{1}$ and $u_{2}$ are positive solutions of $u^{\prime \prime}+\lambda m_{0} u=0, u(0)=u(1)=0$, and we obviously must have $\lambda m_{0}=\pi^{2}$.

Q.E.D.

The following theorem is an immediate consequence of Lemma 3.1 and the results of the preceding section.

Theorem 3.1. Let $f(w) / w$ be a non-increasing function of $w$ for $0<w<$ $r=+\infty$, and let $m_{0}=\lim _{w \rightarrow 0+} f(w) / w$ (so that $0<m_{0} \leqq+\infty$ ) and $m=$ $\lim _{w \rightarrow+\infty} f(w) / w$ (so that $\left.0 \leqq m<+\infty\right)$. Then $\lambda_{f}\{\rho\}$ is a non-decreasing function of $\rho$ for $0<\rho<+\infty, \lim _{\rho \rightarrow 0} \lambda_{f}\{\rho\}=\pi^{2} / m_{0} \geqq 0$, and $\lim _{\rho \rightarrow+\infty} \lambda_{f}\{\rho\}=$ $\pi^{2} / m \leqq+\infty$. If $0<m_{0}=m<+\infty$, (1.1) has infinitely many positive solutions $\alpha \sin \pi t, \alpha>0$, for $\lambda=\pi^{2} / m_{0}$, and no positive solutions for other values of $\lambda$. If $0 \leqq m<m_{0} \leqq+\infty$, then (1.1) has no positive solutions for $\lambda<\pi^{2} / m_{0}$ or for $\lambda \geqq \pi^{2} / m$, and exactly one positive solution for $\pi^{2} / m_{0}<\lambda<\pi^{2} / m$; there are no positive solutions for $\lambda=\pi^{2} / m_{0}$ unless there is an $r_{0}>0$ such that $f(w)=m_{0} w$ for $0 \leqq w \leqq r_{0}$, in which case (1.1) has infinitely many positive solutions $\alpha$ sin $\pi t$, $0<\alpha \leqq r_{0}$, corresponding to $\lambda=\pi^{2} / m_{0}$. As the eigenvalues $\lambda$ of (1.1) increase from $\pi^{2} / m_{0}$ to $\pi^{2} / m$, the norms of the corresponding solutions increase from 0 to $+\infty$.

Figure 1, in which is sketched a graph of the maxima of the solutions of (1.1) against the corresponding values of $\lambda$, illustrates the assertions of Theorem 3.1. The number $r_{0}$ may be zero, positive, or infinite; if finite, it is assumed to be chosen so that $f(w) \neq m_{0} w$ for $w>r_{0}$. If $f(0)>0, r_{0}$ is zero and $\pi^{2} / m_{0}$ is zero.

Lemma 3.2. Suppose that either $f(0)=0$ and $f(w) / w$ is a non-decreasing function of $w$ or $f(0)>0$ and $f(w)$ is convex in $w$ for $0<w<r$. If, for any fixed $\lambda$, (1.1) has non-negative solutions $u_{0}, u_{1}, u_{2}$, with $\max \left\{\left\|u_{0}\right\|,\left\|u_{1}\right\|,\left\|u_{2}\right\|\right\}<r$, then at least two of these are equal unless $f(w)=\left(\pi^{2} / \lambda\right) w$ for $0 \leqq w \leqq \max \left\{\left\|u_{0}\right\|\right.$, $\left.\left\|u_{1}\right\|,\left\|u_{2}\right\|\right\}$. 


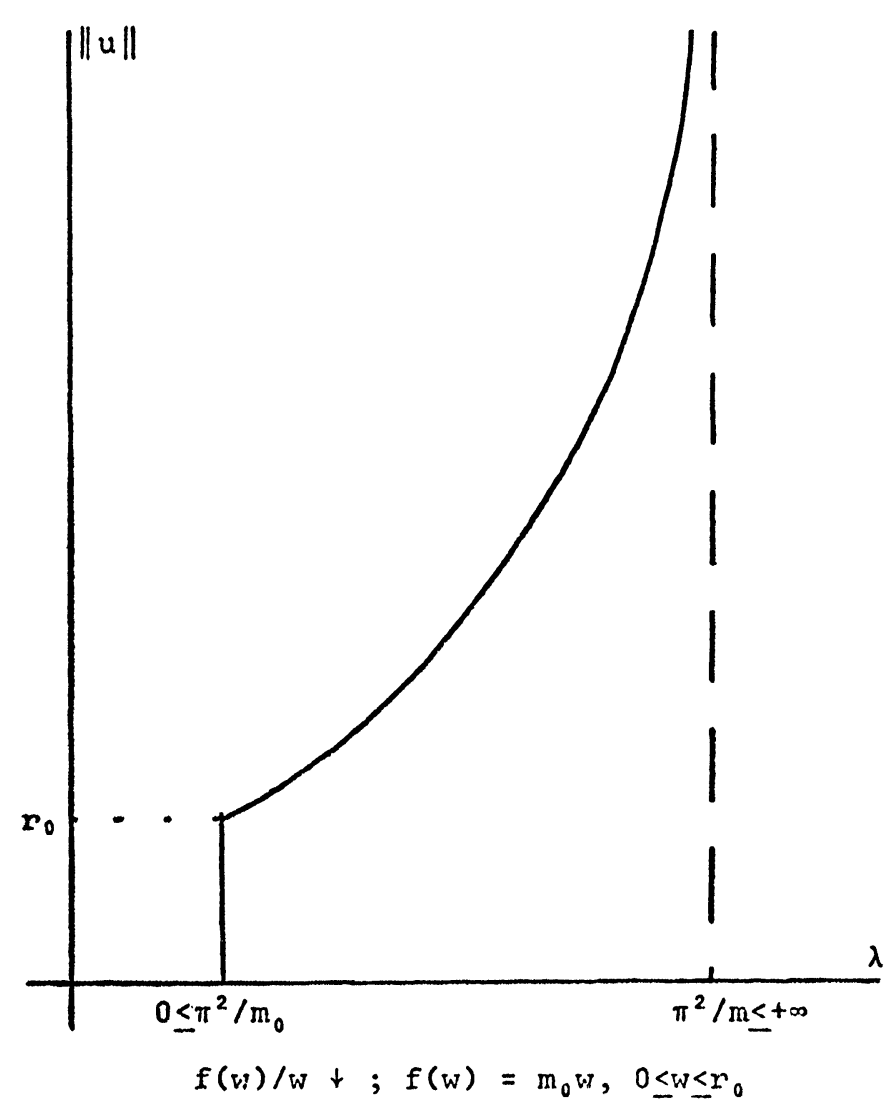

Figure 1.

Proof. The case $f(0)=0$ is handled as in Lemma 3.1. If $f(0)>0$ and there are three positive solutions $u_{0}, u_{1}, u_{2}$, then by Theorem 2.2 we may assume $u_{0}(t)<u_{1}(t)<u_{2}(t)$ for $0<t<1$. The convexity of $f$ implies that $f\left(u_{0}(t)+h\right) / h$ is a non-decreasing function of $h$. Reasoning as in the proof of Lemma 3.1 with $u_{1}-u_{0}$ and $u_{2}-u_{0}$ in place of $u_{1}$ and $u_{2}$, respectively, we find that we must have

$$
\frac{f\left(u_{1}(t)\right)-f\left(u_{0}(t)\right)}{u_{1}(t)-u_{0}(t)}=\frac{f\left(u_{2}(t)\right)-f\left(u_{0}(t)\right)}{u_{2}(t)-u_{0}(t)},
$$

for all $t$ with $0<t<1$. Using Lemma 2.1 and arguments similar to those of Lemma 3.1, we conclude that $f$ must be linear, say $f(w)=m w+b$ with $b>0$ since $f(0)>0$. But then it is well-known that (1.1) has at most one positive solution for each $\lambda$ (the uniqueness also follows from Lemma 3.1). Thus there cannot be 3 solutions $u_{0}<u_{1}<u_{2}$, and the theorem has been proved.

Lemma 3.2 and Theorem 2.12 imply. 
Theorem 3.2. Let $f$ be as described in the first sentence of Lemma 3.2 for $0<w<r=+\infty$, and let $m_{0}=\lim _{w \rightarrow 0+} f(w) / w\left(0 \leqq m_{0} \leqq+\infty\right)$ and $m=$ $\lim _{w \rightarrow+\infty} f(w) / w(0 \leqq m \leqq+\infty)$. If $f(0)=0$, then $m_{0}<+\infty, 0<m, m_{0} \leqq m$, and $\lambda\{\rho\}$ is a non-increasing function of $\rho$ for $0<\rho<+\infty$ with $\lim _{\rho \rightarrow 0} \lambda\{\rho\}=$ $\pi^{2} / m_{0} \leqq+\infty, \lim _{\rho \rightarrow+\infty} \lambda\{\rho\}=\pi^{2} / m \geqq 0$. If $m_{0}=m,(1.1)$ has infinitely many positive solutions $\alpha \sin \pi t, \alpha>0$, corresponding to $\lambda=\pi^{2} / m_{0}$, and no positive solutions for other values of $\lambda$. If $m_{0}<m,(1.1)$ has no positive solutions for $\lambda \leqq$ $\pi^{2} / m$ or $\lambda>\pi^{2} / m_{0}$ and exactly one positive solution for $\pi^{2} / m<\lambda<\pi^{2} / m_{0}$; there are no positive solutions for $\lambda=\pi^{2} / m_{0}$ unless $0<m_{0}<+\infty$ and there is an $r_{0}>0$ such that $f(w)=m_{0} w$ for $0 \leqq w \leqq r_{0}$, in which case there are infinitely many positive solutions $\alpha$ sin $\pi t, 0<\alpha \leqq r_{0}$, corresponding to $\lambda=\pi^{2} / m_{0}$. As the eigenvalues $\lambda$ of (1.1) increase from $\pi^{2} / m$ to $\pi^{2} / m_{0}$, the norms of the corresponding solutions decrease from $+\infty$ to 0 .

If $f(0)>0$ (so that $f$ is convex), Theorem 3.1 is applicable if $f(w) / w$ is nonincreasing for $0<w<+\infty$. If $f(w) / w$ is eventually strictly increasing, then there is a finite positive number $\rho^{*}$ with $\lambda^{*} \equiv \lambda\left\{\rho^{*}\right\}>\pi^{2} / m$ such that $\lambda\{\rho\}$ is an increas-

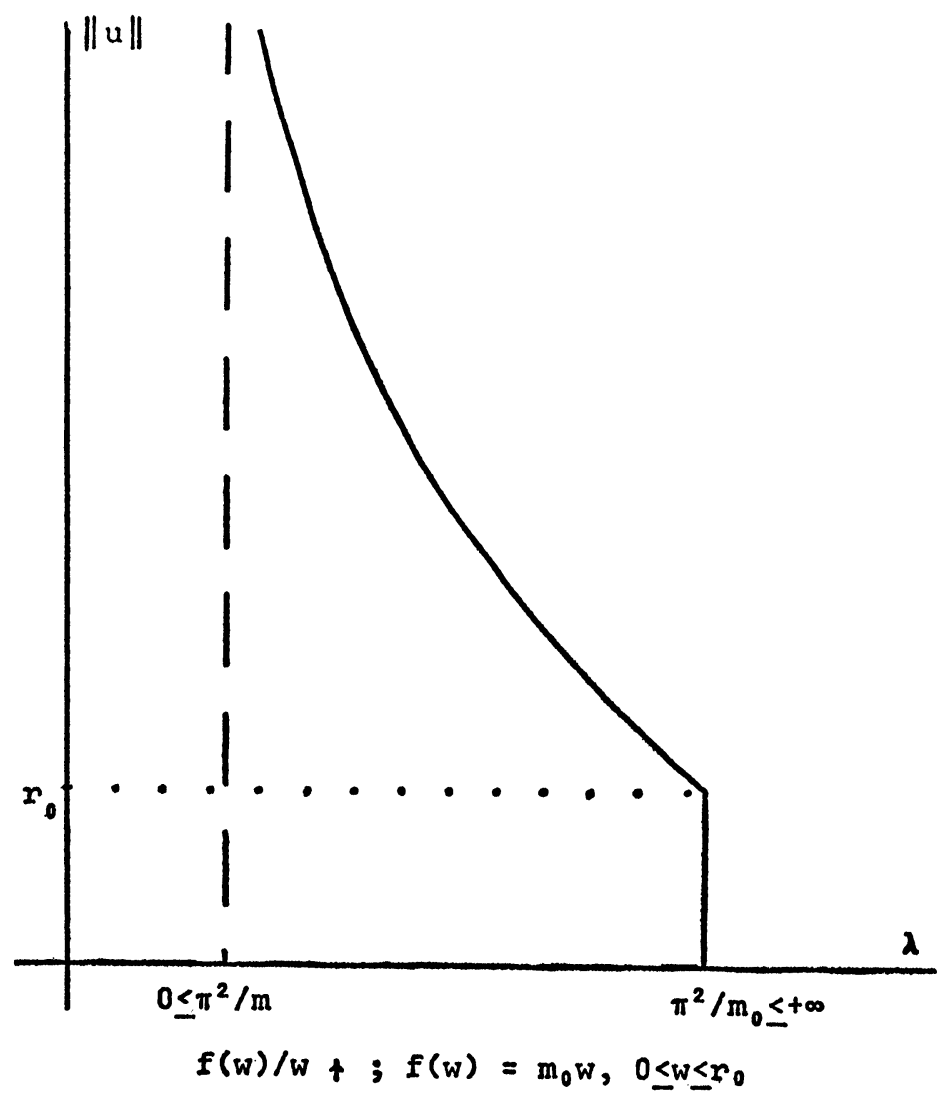

FIGURE 2. 
ing function of $\rho$ for $0<\rho<\rho^{*}$ and a decreasing function of $\rho$ for $\rho^{*}<\rho<+\infty$; $\lim _{\rho \rightarrow 0} \lambda\{\rho\}=0$ and $\lim _{\rho \rightarrow+\infty} \lambda\{\rho\}=\pi^{2} / m \geqq 0$. For $0<\lambda \leqq \pi^{2} / m$ and for $\lambda=\lambda^{*}$, (1.1) has exactly one positive solution; for $\pi^{2} / m<\lambda<\lambda^{*},(1.1)$ has exactly two positive solutions; there are no positive solutions for $\lambda \leqq 0$ and $\lambda>\lambda^{*}$.

Figures 2 and 3 illustrate the assertions of Theorem 3.2.

4. It is clear that, given Theorem 2.2, the proofs of Lemmas 3.1 and 3.2 can be generalized to any self-adjoint operator $L$, in place of the operator $L u=$ $-u^{\prime \prime}$. Using different methods ( $\left.c f .[8, \S 7.1]\right)$, it can be shown that most of the results of Sections 2 and 3 can be extended to multidimensional integral equations of the form

$$
u(x)=\lambda \int K(x, y) f[y, u(y)] d y,
$$

where $K(x, y)$ is a weakly singular kernel, if $f(x, z)$ is positive and monotonically increasing in $z$. However, Theorem 2.2 is apparently not true in general for

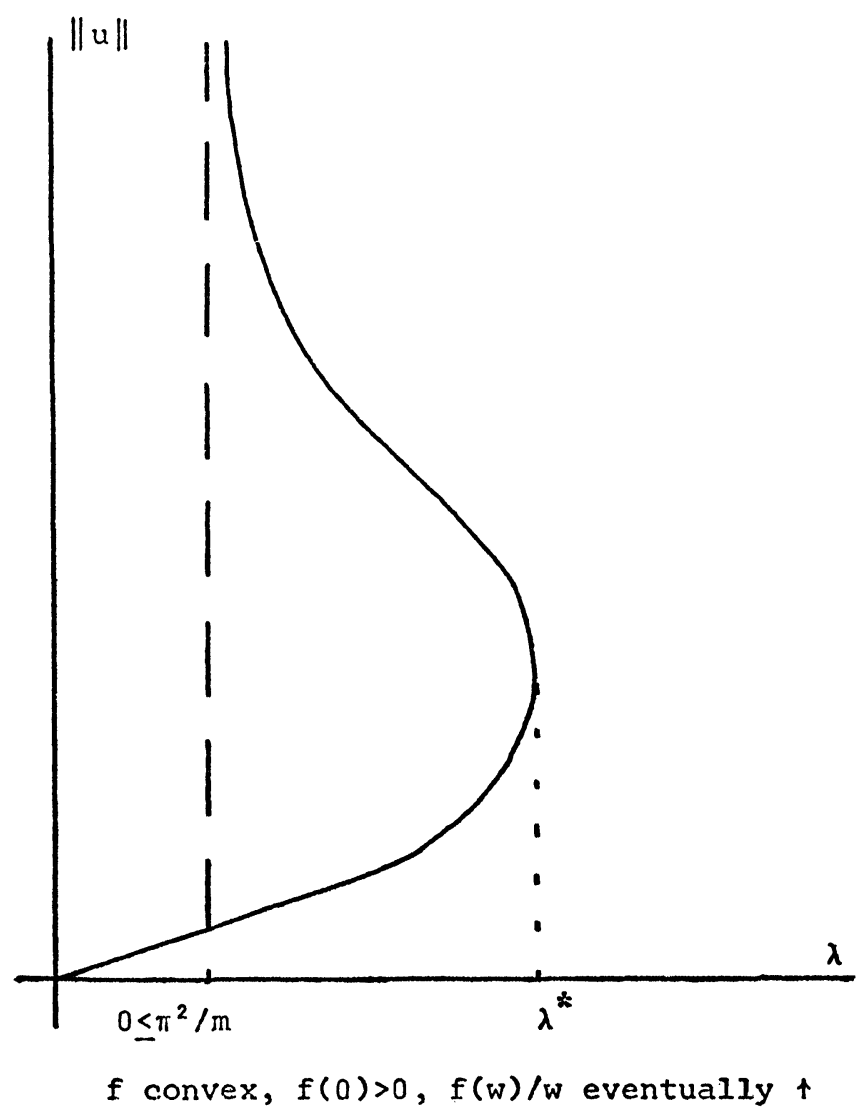

Figure 3. 
(4.1), so the generalization of Lemma 3.2 has the following form: If $f(x, z)$ is convex in $z$, then, for fixed $\lambda$, equation (4.1) does not have three solutions $u_{0}, u_{1}, u_{2}$ satisfying $u_{0}(x)<u_{1}(x)<u_{2}(x)$. The stronger result of Theorem 3.2 on the existence of at most two nonnegative solutions may be extended to differential equations of the form (cf. Moroney [10] and Coffman [3], who treat the case $f(t, 0) \equiv 0)$ :

$$
\begin{aligned}
u^{\prime \prime}(t)+\lambda f(t, u(t)) & =0, \quad 0 \leqq t \leqq 1, \\
\alpha u(0)-\alpha^{\prime} u^{\prime}(0) & =0, \\
\beta u(1)+\beta^{\prime} u^{\prime}(1) & =0,
\end{aligned}
$$

$\alpha, \alpha^{\prime}, \beta, \beta^{\prime} \geqq 0, \alpha \beta^{\prime}+\alpha^{\prime} \beta+\alpha \beta>0$, where either

(i) $f(t, z)$ is independent of $t$, convex, and continuously differentiable, and $\alpha^{\prime} \beta^{\prime}=0$ if $f$ is not strictly convex, or

(ii) $f(t, z)$ is non-increasing (or non-decreasing) in $t$, convex and continuously differentiable in $z, \alpha=0$ (or $\beta=0$, respectively), and, if $f(t, z)$ is not strictly convex in $z, \beta^{\prime}=0$ (or $\alpha^{\prime}=0$, respectively).

Acknowledgment. The author wishes to thank Professor D. S. Cohen for suggesting this problem and for providing valuable advice leading to its solution.

\section{ReFERENCES}

[1] N. Bourbaki, Éléments de Mathématique, IX., Livre IV, Fonctions D'Une Variable Réelle, Actualités Sci. et Ind. 1074, Hermann, Paris, 1953.

[2] P. L. Chambré, On the solution of the Poisson-Boltzmann equation with application to the theory of thermal explosions, J. Chem. Phys., 20 (1952) 1795-1797.

[3] C. V. Coffrman, On the positive solutions of boundary-value problems for a class of nonlinear differential equations, J. Diff. Eq., 3 (1967) 92-111.

[4] Max JАKов, Heat Transfer, Vol. I, John Wiley, New York, 1959.

[5] D. D. Joseph, Non-linear heat generation and stability of the temperature distribution in conducting solids, Int. J. Heat Mass Transfer, 8 (1965) 281-288.

[6] S. A. Kaganov, Establishing laminar flow for an incompressible liquid, translated from the Russian, Int. Chem. Eng., 3 (1963) 33-35.

[7] H. B. Keller \& D. S. Cohen, Some positone problems suggested by nonlinear heat generation, J. Math. Mech., 16 (1967) 1361-1376.

[8] M. A. Krasnosel'skir, Positive Solutions of Operator Equations, translated by R. E. Flaherty, P. Noordhoff, Ltd., Groningen, the Netherlands, 1964.

[9] - Topological Methods in the Theory of Nonlinear Integral Equations, translated by A. H. Armstrong, Macmillan, New York, 1964.

[10] R. M. Moroney, Note on a theorem of Nehari, Proc. Amer. Math. Soc., 13 (1962) 407-410.

[11] G. H. Pimbley, Jr., A sublinear Sturm-Liouville problem, J. Math. Mech., 11 (1962) 121-138.

[12] — A superlinear Sturm-Liouville problem, Trans. Amer. Math. Soc., 103 (1962) 229-248.

[13] M. H. Protter \& H. F. Weinberger, Maximum Principles in Differential Equations, Prentice-Hall, Englewood Cliffs, N. J., 1967. 